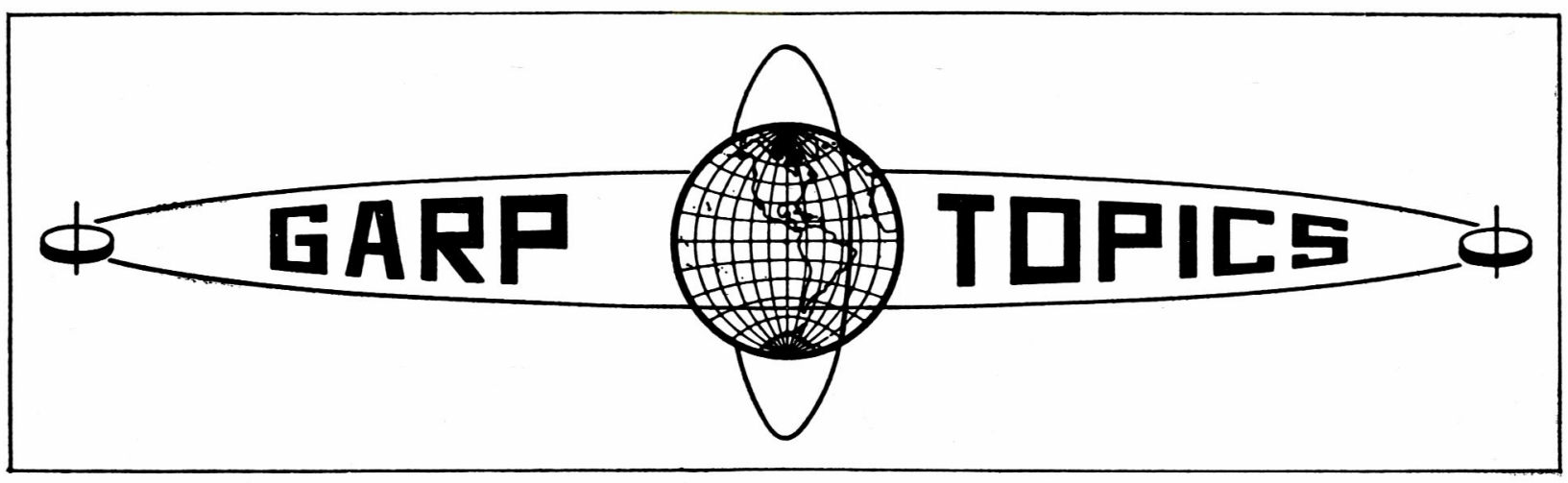

No. 18

October 1971

An occasional series reporting on U. S. and international GARP scientific, technical, and planning activities, developments, and programs, presented as a public service to the meteorological community by the American Meteorological Society through arrangements with the U. S. Committee on the Global Atmospheric Research Program of the National Academy of Sciences-National Research Council. Opinions expressed in GARP TOPICS do not necessarily reflect the point of view of the U. S. Committee.

\title{
Plan for U. S. Participation in the GARP Atlantic Tropical Experiment ${ }^{1,2}$
}

Report of the ad-hoc Tropical Task Group to the U. S. Committee for the Global Atmospheric Research Program, National Research Council

\section{Foreword}

It has been recognized for some time that the meteorology of the tropical oceans constitutes one of the weakest links in our understanding of the general circulation of the atmosphere and therefore in our ability to model weather and climate. Routine synoptic data have never been adequate to define the mesoscale motions, and what detailed knowledge exists has been gained from special observational experiments, the most recent being the Line Islands Experiment, the Barbados Oceanographic and Meteorological Experiment and the Atlantic Trade Wind Experiment. Analysis of the data from such experiments has led gradually to a reliable classification of tropical motions and to preliminary attempts to ascribe mechanisms to the main interactions.

Planning for a definitive tropical experiment has gradually gained momentum from the time of its initial stimulation by UN Resolutions 1721 and 1802-recommending international programs to improve weather forecasting capabilities and advance our knowledge of the physical forces affecting climates-to the present.

1 The activities of the U. S. Committee for the Global Atmospheric Research Program leading to this report have been supported by the National Oceanic and Atmospheric Administration and the National Science Foundation under Contract NSF-C310, Task Orders No. 9 and 197.

2 Available from: U. S. Committee for the Global Atmospheric Research Program, National Academy of Sciences, 2101 Constitution Avenue, N.W., Washington, D. C. 20418.
The Tropical Oceanic Experiment became a major recommendation of the ICSU-IUGG Committee on Atmospheric Sciences and its successor, the WMO-ICSU Joint Organizing Committee for the Global Atmospheric Research Program (GARP). International agreement was reached at the Brussels Planning Conference on GARP, March 1970, to conduct this experiment in the Atlantic Ocean during 1973-1974.

In its first report Plan for U. S. Participation in the Global Atmospheric Research Program, the U. S. Committee for GARP considered an observational study of cumulus-synoptic scale interaction as the most important and demanding of all its recommended field experiments. The central and western equatorial North Pacific was deemed the most suitable region for the experiment. However, when the Committee learned that ground facilities, logistical and satellite support for this area would not become available in the internationally proposed time, the alternative of conducting an experiment in the Atlantic was examined, and it was decided that an Atlantic experiment would be a scientifically justifiable alternative.

Since the publication of the U. S. GARP Committee's plans, analysis of information from the Line Island and BOMEX experiments (particularly the fourth phase of the latter), geosynchronous satellite data, as well as further analysis of earlier observations in the western North Pacific led to a sharpening of the requirements for the Atlantic Tropical Experiment. 
A task group, under the Chairmanship of Richard J. Reed, its former Executive Scientist, was appointed by the GARP Committee to review the new scientific material as well as the documents of a series of international planning conferences which had been held, and to prepare a plan for U. S. participation in the GARP Atlantic Tropical Experiment. The group performed its task outstandingly well, and after careful review by the full U. S. GARP Committee, it has been decided to publish the new plan as an addendum to the earlier publication.

We wish to express our gratitude to Drs. Reed (Chairman), Wallace (Rapporteur), Carrier, LaSeur, Ogura, Riehl, Simpson, Yanai, and Zipser, members of the Tropical Task Group, for the excellent work they have done in preparing the new plan.

\section{Jule G. Charney, Chairman \\ U. S. Committee for the \\ Global Atmospheric Research Program}

\section{Preface}

The development of plans for U. S. participation in the Global Atmospheric Research Program (GARP) has been viewed from the start as an evolutionary process. As scientific objectives of proposed experiments become more precisely defined, as available resources become better specified and as new concepts and constraints are introduced, modification and updating of earlier plans becomes necessary. The present report represents a revised and expanded version of the tropical experiment outlined in the earlier comprehensive Plan for U. S. Participation in the Global Atmospheric Research Program. It takes account of the change of location of the experiment from the Western Pacific area, originally proposed by national and international study groups, to the Atlantic Ocean area and of the international planning activities that have taken place since the issuance of the earlier document.

In June 1970, a group of U. S. experts in tropical meteorology assembled at Miami for a Workshop on Tropical Experiments. This report is based largely on ideas and recommendations developed in sessions held at the Workshop and in the position papers that keynoted the sessions. These papers, and a summary of the proceedings of the Workshop, are available upon request from the U. S. GARP Committee. The plan contained herein is consonant with planning documents issued by ICSU/WMO following the international conference held in Miami (December 1969), Brussels (March 1970), and London (July 1970). Also contributing substantially to the present document were comments and suggestions received from a large number of U. S. scientists in response to a letter of invitation from the chairman of the task group charged with preparation of the report.

The report was drafted by Dr. John M. Wallace with the advice and guidance of the ad hoc Tropical Task Group.

Richard J. Reed, Chairman ad-hoc Tropical Task Force

\section{U. S. GARP recommendations}

The scientific basis for the proposed GARP Atlantic Tropical Experiment has been carefully reviewed by the U. S. GARP Committee. The central unsolved problem for the tropics is the mechanism or mechanisms by which deep cumulus convection is organized in the ITCZ and the synoptic-scale convergence zones, waves and vortices over the tropical oceans. Because of the importance of the resultant release of latent heat in weather and climate this problem is deemed to justify a large-scale effort aimed at describing, analyzing, and explaining the phenomena. It is essential that the observational experiment be carried out in such a manner that the underlying physical mechanisms be exposed and defined. For this purpose certain minimum observational requirements have been stated. These requirements are in part restated below as drawn from Sections 3 and 4 of this report, and certain extensions of the basic requirements are set forth as matters to which additional major attention should be given.

1. The program plan of the GARP Atlantic Tropical Experiment (GATE), having the scientific objectives as described in the report, and as stated in related JOC documents, should be carried out.

2. The GARP Atlantic Tropical Experiment should be carried out during the months of June, July and August 1974, in the Eastern Atlantic. The precise location will be dependent upon logistic considerations and the number of satellites, vessels and aircraft which actually become available.

3. A geostationary satellite having both day and night imaging capability is an indispensable element of the observing network. A daytime-only imaging capability would seriously compromise both the scientific and operational effectiveness of the experiment.

4. Adequate observations need to be made to define the ITCZ and wave scale phenomena. Satellite viewed cloud motions will be helpful in defining the wind field at the trade wind cumulus and cirrus levels. Polar orbiting satellites with IR sounding capability will be adequate for defining the major synoptic features in subtropical regions. In addition, it will be necessary to have a certain number of upper air wind soundings in the tropical regions. These might either be conventional sondes released from ships, or dropsondes released from high flying aircraft. The extent of this large-scale network has yet to be determined.

5. Monitoring of the bulk properties of convective ensembles will require a closely spaced array of ships with a full sounding capability and calibrated radar. (Ideally, one might prefer to look at the whole network with one calibrated radar-see item No. 10.) 
Intensive monitoring of the sub-cloud layer will require special instrumentation, such as the tethered balloon, which must be able to take observations during periods of disturbed weather. Observations of the state, temperature, and radiation budget of the sea surface should also be made from the ship-based network.

6. The number of aircraft pledged at the Brussels meeting must be increased in order to ensure that the experiment will obtain representative samples of the life history, distribution, internal structure, and immediate environment of convective elements. It will be necessary to increase the number to approximately 10-12 aircraft if the performance and instrumentation of the additional aircraft are comparable to those pledged at Brussels. In order to follow the full life history of convective ensembles, some nighttime observations will be needed.

7. It would be desirable to have IR temperature soundings from geostationary satellites, even if only on an experimental basis, since these would supply unique temperature and moisture data continuously in time. In addition, this IR sounder would complement the polar orbiting IR sounders that are required for the First GARP Global Experiment (FGGE) in 1976.

8. Supplementary observations or tests from research satellites might be scheduled to occur during the period of the GATE. Among these observations are superpressure balloon experiments, wind profiles from carried balloon dropsondes, moisture and temperature observations using the microwave technique from polar orbiting satellites and high resolution stereophotography from manned space flight and Earth Resource Survey Satellites.

9. In view of the full rawind capability from ship platforms being a critical observational requirement, the U. S. and other countries should make urgent efforts toward further development and trial of upper air wind-measuring systems based on navigational aids. These results should be made generally available as soon as possible.

10. Studies on the problem of estimating rainfall rate from a combination of radar data, enhanced satellite photographs and raingage records should be undertaken. The feasibility of making these observations from geostationary altitudes should be critically examined.

11. Tests of the GATE and FGGE data systems should be conducted well before the operational phases of these programs to ensure that the systems will support the planned objectives.

\section{Introduction}

\subsection{The GARP Tropical Subprogram}

In GARP publication No. 4 (Sec. 1.1.1) the aims of the GARP Tropical Sub-Program are summarized as they relate to the main objectives of GARP.
The GARP Tropical Sub-programme is primarily concerned with the problems related to the energy-exchange processes between the various scales of atmospheric motions in the tropical atmosphere. It is hoped that an understanding of these processes will lead to finding ways of representing them in terms of parameters defined by the large-scale variables. Hence, the basic purpose of the GARP Tropical Subprogramme is to study those physical problems in tropical meteorology, solutions to which are deemed to be essential for the development of adequate numerical models of the large-scale atmospheric circulation.

A number of tropical meteorologists have emphasized that the problems of forecasting in the tropics deserve attention in their own right and not only insofar as they relate to the problem of middle latitude forecasting.

Fortunately, there is no need to debate this issue, since regardless of the motivation, an improved understanding of scale interaction processes in the tropics will serve both ends. Knowledge of tropical motion systems will serve to improve the accuracy and usefulness of numerical prediction models for the tropics as well as for middle latitudes.

\subsection{GARP experiments}

GARP Report No. 3 (Sec. 1.2.1) defines GARP Experiments: as "consisting of large observational programs designed to determine the behavior of the whole atmosphere or some part of it relevant to the particular sub-program." This definition specifically excludes field programs of a purely exploratory nature, since each experiment should be designed to accomplish a specified set of scientific objectives. While it is recognized that it may be desirable to formulate these objectives in terms of one or more specific hypotheses to be tested, as is often the case in laboratory experiments, a field program of the extent here contemplated could not be justified if classification of tropical motion systems and preliminary physical hypothesis-making had not progressed to a point where a high probability of defining the essential physical mechanisms did not exist.

\subsection{Characteristics of the Atlantic Experiment}

The factors which determine the nature of the GARP tropical experiments are mentioned in GARP Publication No. 4 (Sec. 1.1.2b):

In the design of each experiment a delicate balance must be reached between scientific requirements based on theoretical considerations and previous observational findings, on the one hand, and the practical constraints imposed by the availability of technical means and financial resources, on the other.

In the following paragraphs we will give a broad outline of the scientific requirements and the practical constraints which relate to this experiment. Section 3 outlines an observational strategy which reflects the desired balance.

1.3.1 Scientific requirements. There is a broad consensus among meteorologists in this country that the 
experiment should focus upon the interaction between synoptic disturbances and organized, deep cumulus convection, $^{3}$ and the mechanism responsible for the Intertropical Convergence Zone. Section 2 considers the question of scientific objectives in some detail.

1.3.2 Practical constraints. The experiment is planned to take place in the tropical north Atlantic during the summer of 1974, over a period of about three months. The region of the experiment will be under surveillance by one or more geostationary satellites plus a number of polar orbiting satellites. At the Brussels meeting in March 1970, the participating nations tentatively pledged a total of between 15 and 24 ships for the experiment, most of which would be fully equipped for meteorological and oceanographic observations. Four of these were pledged by the United States for fulltime use and a fifth for part-time use. The U. S. also pledged the use of three research aircraft. However, two of these would be available only when not needed for hurricane reconnaissance work. The USSR pledged one or two specially equipped long range aircraft, and the UK pledged one aircraft specially equipped for cloud physics investigations. Canada indicated that it might be able to provide one specially equipped aircraft. Further details regarding special facilities available for the experiment are given in the Brussels Report as summarized in the GARP Special Report No. 1.

\section{Scientific objectives}

The problem of the interaction between synoptic and convective scale motions may be viewed in two different ways. One might seek a complete physical understanding of the mechanisms by which the scale interaction takes place. This is the approach of the physical scientist, or the numerical modeler who is interested in convection for its own sake. On the other hand, one might be satisfied with parameterizing 4 the collective effects of con-

\footnotetext{
3 In the terminology of GARP Report No. 4, it is desired to study the interaction between Scale $A$ and Scales $C$ and D. This represents a departure from the experimental strategy adopted in GARP Report No. 4 (Sec. 3.1) and subsequent JOC reports. In these reports there is considerable emphasis on the "cloud cluster" scale of 100-1000 km (Scale B). Two types of experiments are defined: Type I which deals with the interactions between Scales A and B and Type II which deals with the interactions between Scales B and C, including the collective effect of Scale D. It is firmly held that the cloud cluster scale can only be defined in terms of the other scales (i.e., the cloud cluster is the rain area of synoptic scale disturbances, and it is the envelope of active cumulus convection). It does not represent a scale on which atmospheric instabilities occur in the same sense that the convective and synoptic scales do. Thus, no particular significance is attached to the interaction between Scale $B$ and the other scales. Type $I$ and Type II experiments cannot be wholly separated.

4 "Parameterization" is a simplification introduced into a dynamical model by preassigning the magnitude of a physical effect rather than allowing the effect to be realistically determined internally as a consequence of the dynamics of the system.
}

vective scale motions upon the synoptic scale disturbances in terms of the synoptic scale fields, without understanding the detailed structure of the convection. This attitude is typical among numerical modelers who are primarily concerned with predicting the evolution of the synoptic scale fields, and is the view point prevalent in the GARP planning documents. The first approach requires detailed knowledge of the internal structure of convection and how it responds to changes in the synoptic scale environment, while the second approach is mainly concerned with relating the bulk properties of convective ensembles to the synoptic scale fields.

Both approaches are consistent with the overall objectives of GARP, yet each suffers from inherent shortcomings. The mere task of documenting the various types of mesoscale organization which convection is capable of assuming could easily absorb all the efforts of many GARP experiments. The prospect of fully understanding the dynamical mechanisms responsible for each type of organization, and how they depend upon the synoptic scale environment is indeed remote. Only through recourse to the bulk properties of convective ensembles can one avoid becoming hopelessly mired in the complexities of convective scale phenomena. On the other hand, complete reliance on parameterization techniques is bound to lead to unfounded empiricism and ultimately to stagnation. It would appear that the best way of avoiding the pitfalls of the two approaches is not to rely on either one of them entirely. Here, as in the field of atmospheric turbulence, a coordinated effort to understand both the internal structure, and the bulk properties represents the path to progress.

Thus, an experiment is envisioned that will provide simultaneous measurements of:

a) the synoptic scale fields,

b) the internal structure of convective ensembles, and

c) the bulk properties of convective ensembles.

The scientific objectives of the experiment can be expressed in terms of the interrelations between these three sets of measurements:

(a-b) to relate the internal structure of convective ensembles to the synoptic scale environment (see also Fig. 1).

(b-c) to explain the bulk properties of convective ensembles in terms of their internal structure, and

(c-a) to use the bulk properties of convective ensembles to parameterize the effects of convection on the synoptic scale fields in terms of the synoptic scale variables.

These objectives will not be realized until the results of the experiment are fully analyzed.

The remainder of this section is to be divided into two parts. First, Section 2.1 briefly reviews the present state of knowledge of (a), (b) and (c) in Fig. 1, as it relates to the tropical Atlantic. Section 2.2 summarizes what is known concerning the interrelationships between $(a-b),(b-c)$ and $(c-a)$. Wherever possible, questions are posed which should be answered by the experiments. 


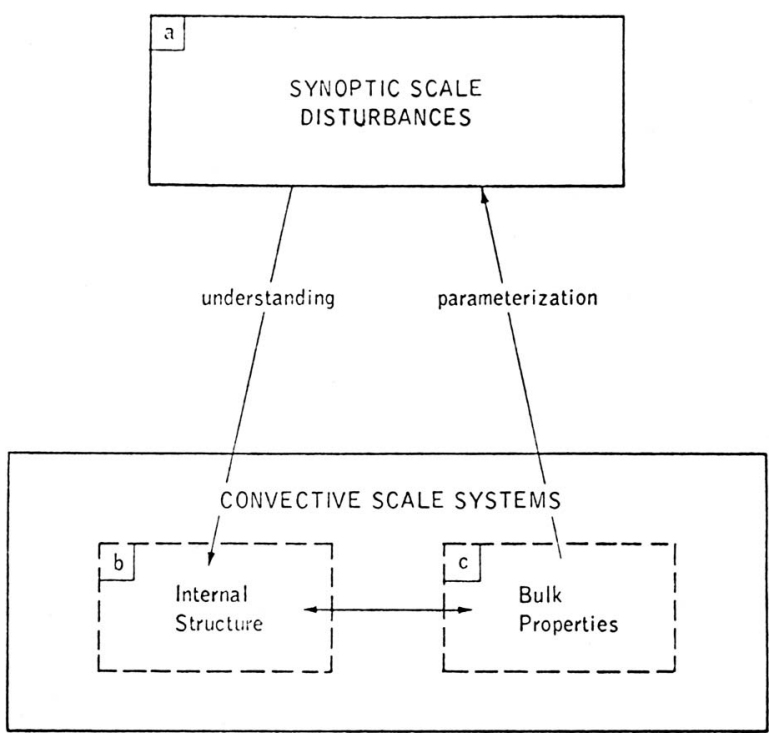

FIG. 1. Schematic relationship between the synoptic scale field and the bulk and internal characteristics of the convective scale field.

\subsection{Properties of the observed fields}

a) Synoptic scale systems in the Atlantic. It is generally agreed that Atlantic Systems are somewhat weaker than their counterparts in the tropical northwest Pacific; wind fluctuations are generally smaller, and rainfall amounts are typically about half of those recorded along the Pacific ITCZ. Nevertheless, there is evidence of definite synoptic scale organization in the Atlantic systems and considerable day to day continuity. In fact, it appears that many of the Pacific systems can be traced backward across the Atlantic to an origin over Africa.

Synoptic systems of the tropical North Atlantic summer season have been classed in four categories:

1) The Intertropical Convergence Zone (ITCZ) which marks the boundary between the northeast trades and low-level, cross-equatorial flow. It is well marked in the eastern Atlantic where it lies close to $10 \mathrm{~N}$. It is usually concentrated in a narrow band 100 to $300 \mathrm{~km}$ in width, comprising one or more lines of active mesoscale convection. It undergoes frequent distortion due to the passage of wave disturbances.

2) ITCZ disturbances, which enter the Atlantic at about $10-12 \mathrm{~N}$ and track west-southwestward to about $8 \mathrm{~N}, 45 \mathrm{~W}$, at a rate of about six degrees of longitude per day. Near this point about two-thirds of these systems lose their identity while the remaining third re-intensify and move northwestward through the Antilles, where some of them reach tropical storm intensity. The cloud patterns associated with the ITCZ disturbances are often vortical in shape, and show evidence of deep convection.

3) Tradewind disturbances, which cross the Atlantic at latitudes near $15 \mathrm{~N}$, at a rate of about six degrees of longitude per day. These are marked by the characteristic "inverted V" cloud patterns which are believed to consist largely of low clouds. These systems exhibit maximum amplitude in the middle troposphere (about $600 \mathrm{mb}$ ), and have relatively little surface weather associated with them.

4) Upper tropospheric vortices which form in association with the tropical upper tropospheric trough. (The latter is a recurrent feature of the summer circulation, extending from the Azores south-westward to the Caribbean.) The more intense vortices extend through much of the depth of the troposphere and exhibit characteristic "comma shaped" cloud patterns.

When the westward moving ITCZ and tradewind disturbances come under the influence of the upper tropospheric trough, in the region of the Antilles, a sudden flareup of convective activity is often observed.

At the present time, considerable uncertainty exists regarding the structure and energetics of all three types of disturbances mentioned above and the nature of the interactions which take place between them because of the lack of radiosonde data. There is also serious question as to whether satellite data alone will be sufficient for defining these disturbances over vast regions of the Atlantic where radiosonde coverage is not available.

b) Internal structure of convective ensembles. The limited data presently available suggest the following view of the internal structure of convective ensembles. Within regions of disturbed weather, active convection is confined to around ten per cent of the surface area. Much of the remaining area is cloudy, but this cloudiness is associated with inactive cloud debris which produce little measurable rain. The active convection sometimes takes the form of isolated cells; at other times it is organized into lines, rings, or horseshoe shaped patterns which have meso. scale dimensions. Saturated updrafts occupy only about ten per cent of the area of active convection (about one per cent of the regions of disturbed weather). The remaining $90 \%$ is divided between a small area of strong saturated downdrafts, and a larger area of weak, unsaturated downdrafts which occupy most of the rain area. Updraft and saturated downdraft speeds are believed to be on the order of 1-10 m sec ${ }^{-1}$; unsaturated downdraft speeds, perhaps a few tenths of a m sec ${ }^{-1}$. Updrafts are strong enough to carry liquid water well above its condensation level, into the upper, anvil shaped portion of the clouds, where vertical wind shear disperses it over a wide area. This explains why the rain areas are large, compared to the updraft areas. A portion of the rain evaporates as it passes through relatively dry, midtropospheric air, cooling the latter and causing it to become more dense than its environment, hence the downdrafts.

Updrafts consist of air originating in the subcloud layer. Despite their relatively small dimensions 
(updraft radii in excess of $2 \mathrm{~km}$ have never been observed), there is strong evidence that the inner cores of the updrafts do not mix with their environment. For this reason, they have often been referred to as "undilute hot towers." The actual temperature excess of the updraft over its environment has been estimated in various studies to lie between 0.1 and 10C. There are strong thermodynamic arguments for placing it in the range from $2-5 \mathrm{C}$ at the $500-\mathrm{mb}$ level. Temperature excesses of this magnitude could produce updrafts on the order of $100 \mathrm{~m} \mathrm{sec}^{-1}$ or more were it not for the weight of liquid water and the retarding influence of form drag. Downdrafts have been measured to be on the order of $\mathrm{IC}$ cooler than their environment with the largest anomalies occurring near the ground.

In well developed mesoscale convective systems there is a strong concentration of cyclonic vorticity in the low level wind field at the base of the updraft. Values on the order of $1-5 \times 10^{-3} \mathrm{sec}^{-1}$ have been measured by aircraft on a number of occasions. These large vorticities are accompanied by horizontal convergences of comparable magnitude. At the levels of cirrus outflow (about $250 \mathrm{mb}$ ) vorticity and divergence, as inferred from ATS cloud motions are opposite in sign from those at low levels and are diffused over a larger area.

Gusty surface winds and cool temperatures associated with downdrafts cause a local enhancement of sensible heat flux from the sea surface, which results in a rather rapid modification of the downdraft air.

Organized cloud groups apparently originate when the low-level mixed layer attains a thickness such that it extends above the condensation level of the air within it, over a distance roughly $10 \mathrm{~km}$ on a side. Small cloudlets, $100-300 \mathrm{~m}$ in radius, break out where the moisture subcloud eddies reach their condensation level. In undisturbed weather, time-lapse pictures have shown that cloud groups often move much more slowly than the wind, sometimes remaining nearly stationary over or just downwind of a sea surface warm spot. Individual cloudlets form on the upwind side of the group and perish as they become the last cloud on the downwind side.

Relatively little is known concerning the variations in the internal structure of meso-convective elements over their life cycles, particularly during the development stages. Of particular interest is the question of whether large low level vorticities are present initially, or whether these develop as a result of conservation of circulation during a prolonged period of boundary layer convergence. It will also be important to establish the "relaxation time" over which downdraft air is modified until it assumes typical boundary layer characteristics.

c) Bulk properties of convective ensembles. They have been studied independently by a number of investi- gators using compositing and spectrum analysis techniques on radiosonde data from the western Pacific. Many of the following statistics represent averages of many individual systems obtained from these studies.

Disturbed weather tends to occur near or slightly east of the low level troughs in "cloud clusters" with dimensions on the order of 2-10 degrees of latitude on a side. Cloud clusters represent local maxima in cyclonic vorticity (about $10^{-5} \mathrm{sec}^{-1}$ ) and convergence (about $5 \times 10^{-6} \mathrm{sec}^{-1}$ ) in the lower tropospheric flow, and anticyclonic vorticity (about $10^{-5} \mathrm{sec}^{-1}$ ) and divergence $\left(1-2 \times 10^{-5} \mathrm{sec}^{-1}\right)$ at the 200 -mb level. The low level convergence of mass and moisture is spread through a rather deep layer of the lower troposphere, with only about $30 \%$ of the mass and about $50 \%$ of the moisture being supplied by the sub-cloud layer.

In the area average disturbed regions are cool in the lower troposphere, warm near the 300 -mb level and cool at the tropopause, relative to their surroundings. Temperature anomalies are on the order of $1-2 \mathrm{C}$ at the surface and $0.5-1.0 \mathrm{C}$ at the higher levels. Above the sub-cloud layer relative humidities are $10-30 \%$ higher than in undisturbed regions but are still well below saturation. (Within the sub-cloud layer there is very little humidity difference between disturbed and undisturbed conditions.) Maximum rising motion occurs near the 300 -mb level where it reaches about $3 \mathrm{~cm} \mathrm{sec}^{-1}$. This is consistent with observed rainfall rates on the order of a few $\mathrm{cm}^{\text {day }}{ }^{-1}$.

Most of the above statistics represent broad averages. It would be highly desirable to know how much variability there is from one cloud cluster to another, and over the lifetime of an individual cluster. It will also be of prime importance to determine how much information the satellite can provide regarding the bulk properties of convective ensembles.

\subsection{Interrelations between the observed fields}

(a-b) The synoptic scale environment could influence the intensity and internal structure of convection in at least three different ways:

1) It has been proposed that variations in synoptic scale static stability exert a controlling influence on organized convection in the tropics. Although in a general sense, convection can be viewed as a consequence of the destabilizing influences on the tropical atmosphere, it does not necessarily follow that convection must take place in those regions where the static stability is lowest. In fact, the reverse appears to be true: regions of disturbed weather are observed to be more stable than their surroundings, as a result of the stabilizing influence of convection that has already taken place. Apparently it is organized convection which controls the synoptic scale 
distribution of static stability, 5 and not the reverse.

2) It has long been recognized that low level convergence is a necessary condition for the maintenance of deep, penetrative convection which is characteristic of disturbed weather in the tropics. When this ingredient is absent, the size spectrum of cloud sized exhibits a logarithmic distribution, with very few, if any, clouds reaching radii of $1.5-2.0 \mathrm{~km}$ (which appears to be the minimum size required to penetrate, undiluted, through the middle troposphere). In the absence of low level convergence, clouds reach heights of only 3-6 km, and very little precipitation reaches the ground. However, when low level convergence is present, the size distribution becomes highly bi-modal, with just the very large and very small clouds present. Middle size clouds are thought to be suppressed by the stabilizing influence of the large clouds, whose circulations replace the ambient subcloud layer air with cooler, drier downdraft air.

But here again we have a "chicken and egg" problem, for it might equally well be said that the presence of deep cumulus convection is a necessary condition for strong synoptic scale divergent motions at any level in the tropics. This has been shown to be a consequence of scaling considerations. It has been hypothesized that the mutual dependence of the synoptic scale divergence field and the distribution of deep cumulus convection in the tropics may, under certain conditions, provide a growth mechanism for synoptic scale perturbations. This phenomenon is called conditional instability of the second kind (CISK) to distinguish it from ordinary conditional instability which gives rise to cumulus scale motions.

When stated in this way, the CISK hypothesis is irrefutable. The problem is that in order to make use of this concept in numerical prediction models, it is necessary to make additional assumptions in order to relate the sub-cloud layer convergence to the synoptic scale motion field and to parameterize the collective effects of the convection upon the synoptic motions. At present, we are lacking a sound physical basis for these assumptions. We will return to this problem when we discuss the interrelation between the bulk properties of convection ensembles and the synoptic scale fields.

3) It also seems likely that the synoptic scale vertical wind shear should have an influence, if not on the amount of convection, at least upon its internal structure and upon the type of mesoscale organization that it assumes. For example, it is observed that strong vertical

\footnotetext{
5 Variations in parcel static stability are actually very small.
}

wind shear is a necessary condition for the development of severe convective storms in middle latitudes.

4) Water temperature through wind driven mixing.

(b-c) The bulk properties of convective ensembles can be deduced from their internal structure, and they can also be measured directly. In this respect, the observational plan for the experiment contains a certain amount of redundancy. However, since considerable uncertainty is inherent in both sets of measurements, this redundancy plays a crucial role in the design of the experiment.

It has been mentioned that much of the convergence of mass and moisture into regions of disturbed weather takes place above the sub-cloud layer. The vertical profile of synoptic scale divergence may represent the superposed effects of three types of internal circulations: buoyant updrafts, which carry sub-cloud air upward to near the 200-mb level; entrainment into updrafts; and evaporatively cooled downdrafts, which replace some of the loss from the sub-cloud layer with air that has entered the region from above.

It appears that the strong concentrations of cyclonic vorticity at the bases of updrafts can easily account for the low level (synoptic scale) vorticity observed in the disturbed regions, even if the remaining some 99 per cent of the area contributes nothing. Because of this tendency for vorticity to be strongly concentrated there may very well be quite large frictional losses of cyclonic vorticity to the ground, even though the synoptic scale low level vorticity is not large. This may explain why, in a bulk sense, there is a flux of cyclonic vorticity into disturbed regions at low levels, and an export of anticyclonic vorticity at upper tropospheric levels.

(c-a) The bulk properties of convective ensembles provide a convenient means of parameterizing the effects of convection upon the synoptic scale motions. Using the data on bulk properties, it is possible to compute the rate of generation of synoptic scale available potential energy by condensation heating, and the rate at which this is converted into kinetic energy. This has been done successfully, using compositing and spectrum analysis techniques, and there is every reason to believe that it can be done for individual cases if the experiment is designed properly. It should also be possible to parameterize the vertical flux of momentum by convective scale motions.

The most difficult task in parameterizing the convective scale motions is to predict the future distribution of convective activity on the basis of synoptic scale information. In the case of the symmetric hurricane, it is possible to obtain an analytic boundary layer solution which prescribes the distribution of low level convergence, which, 
in turn, is assumed to be proportional to the intensity of convective activity. However, in the general case, a boundary layer solution is much more difficult to obtain. Moreover, it is not at all clear that the distribution of convective activity is determined uniquely by frictional convergence in the boundary layer. Some other source of vertical motions may yet prove to be of equal or greater importance.

\subsection{Concluding remarks}

The prospect of the experiment has stimulated a great deal of research in this field. If progress continues at its present rate, it is not unreasonable to expect that in the three years which remain before the experiment, some of the current thinking on the scale-interaction problem will have to be reviewed. This is why in the previous sections, the observational basis for hypotheses has been stressed rather than the hypotheses themselves. And this is why the scientific objectives of the experiment have been expressed in the terms of consolidating and extending our observational knowledge, rather than as a test of some specific hypothesis. In this way, we can assure that the experiment will be highly relevant to the problems existing at the time of the experiment.

At the same time we recognize the importance of hypothesis-making in general. Without hypotheses to be stated, there is no basis for distinguishing among a variety of events of equal a priori relevance. While no one hypothesis is presently capable of explaining the complicated convective phenomena of the tropics, existing hypotheses, such as CISK, at least focus attention on events in the sub-cloud and frictional boundary layers as deserving of concerted observation.

\section{Observations strategy}

To accomplish the stated scientific objectives, the observational network must be capable of:

1) defining the synoptic scale fields over the entire tropical Atlantic and neighboring land masses with sufficient accuracy and resolution to define the major synoptic systems.

2) determining the bulk of properties of convective ensembles.

3) intensive sampling of the internal structure of the convective ensembles whose bulk properties are being monitored, in order to obtain representative statistics.

Each of the above functions will require a special combination of observational tools and techniques. The total observational program can be viewed as consisting of the sum of these three subsystems, plus whatever is needed to coordinate them in order to ensure that the observational requirements of the experiment are met.

\subsection{Definition of the synoptic scale fields}

Definition of the synoptic scale fields will be accomplished primarily by satellite observations which must be routinely available by the time of the experiment.
These will be supplemented by radiosonde observation from land and ship stations in the region of the experiment together with reports from commercial aircraft and ships of opportunity (in addition to the ordinary network, a number of additional stations are being established as part of the World Weather Watch). It is also expected that a number of oceanographic vessels will be making regular traverses of the ITCZ.

A certain number of dropsonde or ship-based radiosonde observations may be required to provide additional definition over the remote mid-oceanic regions. The number of ships which can be used for this purpose will be limited to those which are available over and above what is needed for monitoring bulk properties of convective ensembles (see Sec. 3.2). The extent of the mid-ocean synoptic network is one of the key remaining questions to be settled.

This synoptic network will operate on a fixed schedule in real time. The processing, analysis, and archiving of these data can be accomplished largely through the use of existing operational facilities.

\subsection{Determination of the bulk properties of convective ensembles}

Determination of the bulk properties of convective ensembles will require:

1) Detailed vertical profiles of the convergence of mass, heat, moisture and momentum into the area enclosing one or more such ensembles (radiosonde data).

2) Mapping of the distribution of active convective elements within the area (satellite reconnaissance and radar).

3) Quantitative estimates of total precipitation falling within the area (radar and some raingages).

In order to obtain statistics with sufficient accuracy and representativeness it will actually be necessary to sample the bulk properties of a large number of convective ensembles. This can conveniently be accomplished by establishing one or more shipbased networks.

The operation of this network would not be confined to periods of disturbed weather, since it will also be necessary to compile statistics on the bulk properties of the undisturbed tropical atmosphere. At synoptic observation times, the ship based network will also function as an integral part of the synoptic network.

3.2.1 Location of the ship-based networks must satisfy the following requirements:

a) high probability of encountering active convection associated with synoptic disturbances in varying stages of development.

b) proximity of bases for aircraft operations (see Sec. 3.3), supplies, communications, etc.

The only regions which meet these criteria are the extreme western Atlantic in the vicinity of the Leeward Islands, and the extreme eastern Atlantic between $5 \mathrm{~N}$ and $20 \mathrm{~N}$. The latter region experiences a much higher frequency of active convection. The decision of the 
Tropical Experiment Board (see GARP Special Publication Nos. 1 and 2) that the experiment should be conducted in the eastern Atlantic is endorsed provided that the necessary components of the observing system can be made available in this region.

3.2.2 Spacing of ships in the network will be governed by the following considerations:

a) The probability of encountering active convection is proportional to the area of the network (i.e., the square of the station spacing).

b) It would be highly desirable to have overlapping radar coverage. This limits the spacing to approximately $250 \mathrm{~km}$.

c) Spacings of on the order of $500 \mathrm{~km}$ appear to be most efficient from the point of view of monitoring bulk properties.

It would appear that the effectiveness of the ship based network can be maximized by clustering the ships with radar in the inner part of the network, with spacing of about, or less than, $250 \mathrm{~km}$, and by positioning the ships without radar around the periphery of the network on the order of about $500-\mathrm{km}$ spacing.

3.2.3 The success of the experiment depends critically upon our ability to monitor processes occurring within the sub-cloud layer. In order to obtain the necessary vertical and time resolution it will be necessary to supplement the radiosonde data with soundings made with tethered balloons.

3.2.4 The network should be fixed in space and should operate on a regular schedule for radiosonde ascents (6-hr intervals during undisturbed periods and 2- or 3-hr intervals during disturbed periods).

3.2.5 With the exception of certain radar products required for real time operation of the aircraft (see Sec. 3.3), the data from this network can be processed in the constant-lag mode (see Sec. 5). Special centralized facilities will be required for data transmission, reduction and archiving.

\subsection{Intensive sampling of convective ensembles}

Intensive sampling of the internal structure of convective ensembles should aim at providing representative statistics on the following items, ranked in order of priority.

1) The horizontal distribution of divergence and vorticity at various levels in the vicinity of mesoscale convective elements.

2) Heights, vertical profiles and internal structure of deep, convective clouds.

3) Typical updraft and downdraft velocities and areal coverage at various levels.

4) Lifetimes and typical life-cycles of mesoscale convective systems.

In addition to the above items, it would be desirable to obtain information on:

a) The rate of sensible heat flux and evaporation from the sea surface under disturbed conditions. b) Typical distribution of drop sizes, liquid water content, and relative humidity in and around deep convective clouds.

c) Relations between positions of mesoscale convective elements and sea surface temperature anomalies.

d) Radiative heating rates under various cloud conditions.

e) Effective freezing level for cloud droplets in deep convection.

This subprogram of the experiment will be mainly based on the use of instrumented aircraft, which can be viewed as high speed observing platforms, operating on a flexible schedule determined on the basis of real time satellite and radar information. This will be supplemented by ground or ship-based Doppler radar. The aircraft operations will take place within the ship based network discussed in Sec. 3.2, but the flight paths and schedules need not be coordinated with the radiosonde subprogram. The data will be processed in the constantlag time mode.

The success of this part of the observational program will depend critically upon the number and type of instrumented aircraft which are made available by the participating nations. (See Sec. 4.1.4).

\subsection{Other observations}

Other observations may include oceanographic measurements of various types as suggested by the JOC, ${ }^{6}$ aerosol sampling, and measurements related to cloud microphysics. In general, these observations will be planned, implemented and processed by individual investigators and, therefore, fall outside the range of responsibility of the planning committees, except in cases where they interact with the other subprograms (e.g., in use of facilities). Every effort should be made to accommodate these peripheral observational programs, as long as they do not interfere with the observational plan stated above.

\subsection{An overview}

We have seen that the overwhelming task of formulating a comprehensive observational program can be simplified to some extent by breaking it down into three subprograms, each of which is designed to accomplish a limited set of objectives. Quite fortuitously, it happens that each of the subprograms described above requires a different set of observational tools; apart from the satellite, there is relatively little overlap between the three observing subsystems. It also happens that there exists a precedent for each of the subprograms:

a) Definition of the synoptic scale fields will be based on a refined version of current operational analysis techniques, which already make extensive use of a mix of satellite, radiosonde, commercial aircraft and surface observations.

b) Several groups working independently, have had some degree of success in deducing the bulk properties of

\footnotetext{
${ }^{6}$ Joint (ICSU/WMO) Organizing Committee.
} 
deep cumulus convection on the basis of the island based radiosonde network in the western Pacific. These studies provide a basis for specifying the number of observations, the accuracy and resolution required to obtain adequate definition of the quantities to be measured.

c) Research aircraft have already seen extensive use in past field programs. Published studies based on analysis of the data from these programs have already shown that most of the measurements listed in Sec. 3.3 can be made successfully with aircraft.

Thus, there is a rational basis for the design of each of the three subprograms. During the planning and implementation phases of the experiment, only a minimal amount of coordination between subprograms may be required. It is in the analysis phase that the parts of the experiment should merge into a coherent whole.

For the purpose of clarifying differences between the observational strategy outlined above and those presented in previous reports, it may be helpful to mention a number of objectives which the proposed experiment will not accomplish.

a) It will not be possible to attain a clear definition of equatorial wave modes as envisioned in plans for the western Pacific experiment. Only in the equatorial mid-Atlantic would there be any possibility of studying wave disturbances which are not strongly affected by the land-sea distribution. This area has relatively little active convection and is not conveniently located from a logistical point of view.

b) It will not be possible to resolve individual mesoscale convective systems with the radiosonde network. It is doubtful that this could be done even with a ship spacing an order of magnitude smaller than contemplated in Sec. 3.2.2. A spacing this small would not be compatible with the objective of obtaining definitive statistics on the bulk properties of convective ensembles, since, with the greatly reduced area of the network, it would not be possible to sample a sufficient number of systems during the course of the experiment.

The observational plan outlined above will rely on radar and satellite data for determining the position, size, shape and intensity of the mesoscale convective systems within the network. Since both vorticity and divergence of the horizontal wind field tend to be concentrated within the mesoscale convective elements, there is reason to hope that the area integrals obtained from rather widely spaced stations, if carefully interpreted, can serve to define the gross features of the mesoscale wind field.

c) The experiment is not specifically designed to monitor planetary scale features or long-term periodicities. The synoptic subprogram may provide some useful information on the largest scales, but it should be borne in mind that this will not be much different from the type of information which will be routinely available when World Weather Watch is fully implemented. The experiment will not be of sufficient duration for studying long-term periodicities.

d) A repetition of the detailed study of sea-air exchange processes, which was the central focus of BOMEX, is not recommended. The sea-air transfer measurements mentioned in Sec. 3.3(a) are for the purpose of estimating the typical heat and moisture fluxes from the sea surface during disturbed conditions.

\section{Data acquisition}

In order to carry out the observational plan outlined in the previous section, it will be necessary to deploy a complex and highly integrated observing system. The characteristics of this system have been discussed in some detail in previous reports. In this section we will comment on only those aspects of the observing system which are in need of further emphasis or clarification.

\subsection{Platforms}

4.1.1 The geostationary satellite is an indispensable element of the observational network. Implementation of the experiment should be made contingent upon having at least one such satellite, in fully functioning condition, in orbit over the region of the ground based network. A satellite with capabilities comparable to the proposed SMS/GOES series, including nighttime capability (as compared to the present ATS type) would greatly increase the scientific potential of the experiment. The possibility of IR and radar sounding from geostationary satellite should also be given serious consideration. It would be desirable to have some backup capability in the form of a second geostationary satellite that could be moved into the area of the experiment, should the first one fail.

Polar-orbiting satellites with IR sounding capability for deducing temperature will be helpful in analyzing sub-tropical regions.

4.1.2 The primary function of ships will be to serve as fixed platforms for radar and radiosonde observations. Vessels used for this purpose need not be fully instrumented for research purposes. The network configurations discussed in the previous section will require 8-10 ships on station, continuously, throughout the 3-month period of the experiment.

If the oceanographic research vessels are included as part of the ship-based network, a conflict may arise between meteorological needs, which are best met by maintaining the ships in fixed positions, and oceanographic needs, which require some degree of mobility. A workable compromise can, in all probability, be worked out by allowing these ships to operate freely within some specified radius of their prescribed locations. A radius as large as five per cent of the ship spacing (about $12 \mathrm{~km}$ ), would not present serious difficulties from a meteorological point of view. 
4.1.3 Aircraft will perform two functions in the experiment.

a) Dropping sondes from altitudes about, or higher than $35,000 \mathrm{ft}$ on trans-Atlantic flights. The extent of required dropsonde coverage has not yet been established.

b) Intensive sampling of the internal structure of convective ensembles. This task will require highly instrumented aircraft capable of flying at levels from $500 \mathrm{ft}$ to $55,000 \mathrm{ft}$. Measurements will include Doppler-winds, aerial photography, convective scale vertical motions, temperature, relative humidity, liquid water content, drop size, certain radiative fluxes, vertical fluxes of moisture and sensible heat. To obtain the needed measurements, it will be necessary to have 6-10 aircraft in the air simultaneously during periods of intensive sampling (about 1 day in 4). These will include 2-4 flying below cloud base (these should be equipped with inertial platforms), 1-2 near or above the cloud tops and the remainder at intermediate levels.

A minimum number of 10-12 well instrumented aircraft appear to be required to carry out the intensive sampling outlined in (b). We note that this is about double the number of aircraft presently committed for the experiment!

4.1.4 Tethered balloons show considerable promise as a tool for monitoring the sub-cloud layer. These devices should be placed on as many ships as possible. To be of use in the experiment, the balloons must be developed which are capable of staying aloft during periods of disturbed weather.

4.1.5 Buoys may be of some limited use for surface observations. Highest priority meteorological observations are wind and rainfall. Hourly observations would be sufficient. From a meteorological point of view one additional ship in the network would be of more value than many buoys.

\subsection{Radiosonde observations}

Accuracy requirements.

a) dropsondes:

winds to $2 \mathrm{~m} \mathrm{sec}^{-1}$ in about $150 \mathrm{mb}$ layers; other parameters of less importance.

b) ship based network:

winds to $1 \mathrm{~m} \mathrm{sec}^{-1}$ with vertical resolution to at least $2 \mathrm{~km}$; preferably $1 / 2 \mathrm{~km}$. Temperature to $0.2 \mathrm{C}$. Mixing ratio to $0.5 \mathrm{~g} \mathrm{~kg}^{-1}$.

If the proposed Omega system cannot fulfill the wind requirements, serious consideration should be given to implementing the Loran system over the limited region of the ship based network.

\subsection{Radar}

In past experiments the processing of radar data has lagged so far behind the rest of the data processing that much of the potential of this component of the observational network has not been realized. Since we anticipate that radar will play a major role in the proposed observing system, it is extremely important that this situation be remedied in the time which remains before the experiment.

There is need of a quantitative meteorological radar system with real time, digital data processing. This would reduce vast accumulations of radar film to a manageable amount of digital data which can be integrated with the other results of the experiment. It would also facilitate displays of the distribution of radar echoes over the entire ship-based network, in real time, which would be useful for planning aircraft routing. There is also need for further work in relating radar data to enhanced cloudiness images from the satellite. The latter may be extremely useful for intercalibrating the radar echoes at various distances from the receivers and from different types of equipment.

The problem of deducing rainfall rates from calibrated radar and satellite information deserves immediate attention.

\section{Data processing}

\subsection{Real time requirements}

Those data which are required for the day to day direction and operation of the experiment should be available in real time (elapsed time of a few hours or less) at the experiment headquarters. Only a small fraction of the data from the experiment will be required in the real time mode. This will include cloud images from the geosynchronous satellite (regular and enhanced), certain synoptic analysis products, and possibly some of the radar data from the ship-based network.

In addition it will be necessary to sample the output of the various components of the observing system from time to time, to test whether equipment is functioning properly.

\subsection{Constant-lag time requirements}

Those data which relate to the primary scientific objectives, but which are not required for the real time operation of the experiment, should be processed in the constant-lag time mode. These data should be available to the scientific community, in fully processed form within some specified time interval from the time that they are taken. The time interval is negotiable; it may range from a week to a few months. Data formats should be agreed upon and publicized well before the time of the experiment.

It is probable that a number of investigators will be conducting their own observing programs within the overall framework of the experiment. Data processing requirements for these programs should be left to the discretion of the individuals involved. Data processing facilities should be made available to these investigators on a non-interference basis. 


\section{ad-hoc Tropical Task Group}

Membership

George F. Carrier

Harvard University

Noel E. LaSeur

Florida State University

Yoshimitsu Ogura

University of Illinois

Richard J. Reed, Chairman

University of Washington

Herbert Riehl

Colorado State University
Joanne Simpson

Experimental Meteorology Branch, NOAA

John M. Wallace, Rapporteur

University of Washington

Michio Yanai

University of California, Los Angeles

Edward J. Zipser

National Center for Atmospheric Research

\section{U. S. Committee for the Global Atmospheric Research Program}

Membership

Alfred K. Blackadar

Pennsylvania State University

Jule G. Charney, Chairman

Massachusetts Institute of Technology

John W. Firor

National Center for Atmospheric Research

N. P. Fofonoff

Woods Hole Oceanographic Institution

Richard M. Goody

Harvard University

Cecil E. Leith, Jr.

National Center for Atmospheric Research

Richard S. Lindzen

University of Chicago

Bruce Lusignan

Stanford University

Douglas H. Sargeant, Executive Scientist *

National Research Council

Owen M. Phillips

Johns Hopkins University

Richard J. Reed

University of Washington

Herbert Riehl

Colorado State University

Joseph Smagorinsky, Vice Chairman

National Oceanic and Atmospheric Administration

Henry M. Stommel

Massachusetts Institute of Technology

Verner E. Suomi, Vice Chairman

University of Wisconsin

John M. Wallace

University of Washington

* Through Jan. 1971. Now affiliated with NOAA.
John R. Sievers, Executive Secretary

National Research Council

Ex-officio Members:

Robert F. Fleagle

University of Washington

Thomas F. Malone

University of Connecticut

Invited Participants:

Homer E. Newell

National Aeronautics and Space Administration

Edward P. Todd

National Science Foundation

John W. Townsend

National Oceanic and Atmospheric Administration

Robert M. White

National Oceanic and Atmospheric Administration

Liaison Representatives:

Hugh W. Albers

Department of Defense

Rudolf J. Engelmann

Atomic Energy Commission

Richard E. Hallgren

National Oceanic and Atmospheric Administration

Joseph F. Sowar

Department of Transportation

Morris Tepper

National Aeronautics and Space Administration

Robert T. Webber

Department of State

Fred D. White

National Science Foundation 